\title{
Attentive fields are related to focal and contextual features: A study of Müller-Lyer distortions
}

\author{
ALEXANDER W. PRESSEY and CHRIS A. PRESSEY \\ St John's College, University of Manitoba, Winnipeg, Canada
}

\begin{abstract}
The mathematical model associated with integrative field theory was used to infer the size of attentive fields in a task involving judgments of size. A compounded Müller-Lyer task was employed in which fins between or outside the standard shafts were systematically removed. Performance on this task was simulated by a computer that generated families of theoretical functions varying in the parameter of attentive field size. Individual theoretical functions were then correlated with an empirical function obtained from real observers. The value of attentive field size that provided the best fit between functions was then selected. The results showed that, in almost all cases, the optimum size of the attentive field was smaller for the shrinkage form than for the expansion form of the Müller-Lyer pattern, that the attentive field changed more dramatically with changes in stimulus variables in the expansion form than in the shrinkage form, and that changes in viewing distance had little effect on the optimum size of the attentive field. It was concluded that the attention was involved both in figure-ground segregation and in maintaining object constancy. The similarity of these results to data obtained in recognition and detection tasks was noted.
\end{abstract}

In a perceptual task that requires a response to some attribute involving two or more spatially separated objects, an observer deploys attention in a way that includes those objects. The notion of a field has been proposed to describe such attentional selectivity, and its characteristics have been investigated using tasks involving detection (B. A. Eriksen \& C. W. Eriksen, 1974; Podgorny \& Shepard, 1983; Pressey, Wilson, \& Harper, 1980), discrimination (C. W. Eriksen, Goettl, St. James, \& Fournier, 1989), recognition (Gatti \& Egeth, 1978; LaBerge, 1983; Merikle \& Gorewich, 1979), apparent motion (Gogel \& Sharkey, 1989), and length estimation (Jordan \& Randall, 1987; Pressey, 1974b; Pressey \& Di Lollo, 1978).

It appears that different writers understand the idea of a field of attention in different ways. For example, at a phenomenological level, clarity of the conscious percept has been of primary concern. Wilhelm Wundt and William James (cited in Wertheimer, 1987) observed that what we focus on is much clearer than that which is spatially distant from the focal point. More recently, Mackworth (1965) described a paradoxical effect in which information at the edge of a display exhibits greater conscious clarity than does information that is closer to the focal point (see Figure 1). Finally, C. W. Eriksen et al. (1989)

This research was partially supported by a grant from the University of Manitoba Research Committee of Senate. We are indebted to Bernd Kersten for writing the initial version of the computer program used in the current study and to Tom Holens, who has provided countless impromptu tutorials on the language and constructs of mathematics. Correspondence should be addressed to A. Pressey, St. John's College, University of Manitoba, Winnipeg, Manitoba R3T 2M5, Canada (e-mail: pressey@uofmcc). have used perceptual clarity as a marker variable for observers to initiate a response.

An attentional field has also been used to denote a psychological mechanism that functions to limit the information that is processed (perhaps during a single glance). This idea has been captured by mechanical metaphors, such as moving spotlights (Broadbent, 1982; Posner, Snyder, \& Davidson, 1980) and zoom lenses (C. W. Eriksen \& Yeh, 1985), in which "attentional energy" is allocated to various parts of the field (C. W. Eriksen et al., 1989). These metaphors give rise to the implication that attentional fields are substantive properties of the biopsychic system.

A third way of understanding a field of attention ${ }^{1}$ is to view it as a logical-mathematical construct that refers to a "field of probability" (Pressey, 1979). It is a macrotheoretical construct that describes how information from multiple samples is differentially weighted in forming the (spatially and temporally) integrated output of an intentional system. It is more conducive to an analysis of tasks that span chunks of time in the order of seconds rather than milliseconds-tasks involving comparisons of relative magnitudes or reading text, or esoteric tasks, such as watching reversing Necker cubes and rotating trapezoidal windows. A precedent for such probability fields has been set by postmodern physicists who regard subatomic particles not as substantive entities, but as probability patterns that represent tendencies to be found in various regions of the atom. Jones (1982) has stated,

What the fuzzy-ball representation of an atom shows is not an actual picture of an atom but a sort of time-average of a large number of imprecise position measurements. The idea of a snapshot or instantaneous picture cannot be applied to an atom, not even in principle. (p. 20) 


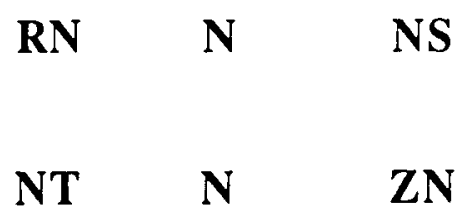

Figure 1. A task that demonstrates conscious clarity. Focusing on the upper $\mathbf{N}$ causes the peripheral $\mathbf{R}$ and $\mathbf{S}$ to be clearer than the inner letters. Focusing on the lower $\mathbf{N}$ causes the two $\mathrm{Ns}$ at the edge to be clearer.

In a similar fashion, it is impossible, in principle, to provide a picture of a "real" attentive field, because such fields are statistical, not substantive.

In spite of the variation in meaning, there is a remarkable similarity in the way in which fields of attention are described. For example, spotlights, zoom lenses, and probability fields all endorse the proposition that such fields are circular although, in some cases, a circular probability field is adopted primarily for pedagogical and quantitative simplicity (Pressey \& Murray, 1976). Now, it is becoming clear that shapes of fields are not universal but are defined, for one, by the spatial configuration of the visual array (LaBerge, 1983). Thus, a field "'might become elliptical, then 'dumbbell' shaped, and then differentiated into two separate circular fields"' (Pressey, 1988, p. 201). Nevertheless, because there is no certain a priori way in which to establish shapes of fields, the circle remains a powerful first approximation for certain conditions.

Fields of attention are also presumed to vary in size. Those who operate at the microstructural level argue that field size is directly related to the size of the focal stimulus (C. W. Eriksen \& St. James, 1986; LaBerge, 1983) and to the size of the contextual stimuli (Gatti \& Egeth, 1978; Merikle \& Gorewich, 1979). Those who utilize temporally extended tasks agree that field size varies with the size of the focal stimulus (Jordan \& Schiano, 1986; Pressey \& Di Lollo, 1978) but deny that attention is captured by contextual as well as focal features. (This problem is the major concern of the present investigation, and we will turn to it shortly). In addition, the size of the probability field is presumed to be a stable personality variable that reflects a preferred style of deploying attention. There is some evidence that attentive field size can be altered by ingestion of alcohol and by sleep deprivation (Pressey, 1982), and it has been shown that including size of hypothetical attentive fields in a simulative model of length distortion predicts not only means, but also variances, of real data (Pressey, Butchard, \& Scrivner, 1971; Pressey \& Murray, 1976).

Finally, it should be noted that many researchers have been concerned with what can be called the "texture of clarity" within a field of attention. The spotlight metaphor leads us to expect information to be equally clear, or equally effective, throughout the entire field. The zoom lens analogy provides for a homogeneously textured field that is tied to field size so that large fields provide low resolution and small fields provide high resolution of detail (C. W. Eriksen \& Yeh, 1985). A macrotheoretical attentive field has much in common with the zoom lens model in regard to resolution. Such fields are presumed to be linearly graded from the center to the periphery. Small fields reflect a high ability to gate out contextual noise that is spatially adjacent to the focal stimulus. Large fields reflect a poor ability to gate out spatially distributed noise and leads to integration (assimilation or contrast) of focal and contextual information (Pressey et al., 1971; Pressey \& Murray, 1976; Pressey \& Wilson, 1980).

\section{ATTENTION IN THE CONTEXT OF AN ASSIMILATION (INTEGRATIVE FIELD) THEORY OF VISUAL DISTORTION ${ }^{2}$}

The idea of an attentive field was developed in the course of an attempt to explain what are known as optical geometric illusions. The most famous of such effects is produced by a Müller-Lyer configuration, versions of which are shown in Figure 4. Inward pointing fins $(<>)$ produce phenomenal shrinkage of the distance between apexes: outward pointing fins $(><)$ produce phenomenal expansion of the distance between apexes.

Drawing from research in psychophysics, perception, memory, and social behavior, it was proposed that individual entities take on the properties of the mean of the context in which they are embedded (Pressey, 1967; Pressey et al., 1971). A corollary of this theorem is that the degree of such "regression to the mean" is directly related to the difference between the individual entity and the mean of the whole. Large differences result in large amounts of change and small differences result in smaller amounts of change.

If this idea applies to a parallel-lines configuration (Figure $2 \mathrm{~A}$ ), the length of a standard stimulus $\left(L_{s}\right)$ will regress to the length of the contextual stimulus $\left(L_{c}\right)$ and appear elongated. Thus, a formula as simple as

$$
\left(L_{c}-L_{s}\right)
$$

describes both the direction of distortion $(+=$ expansion; - = shrinkage) and, more crudely, the degree of distortion in such complex patterns as Müller-Lyer and Poggendorff figures (Pressey, 1972).

It is easy to show that concepts such as "assimilation" or "regression to the mean" are much too simple. For example, Formula 1 predicts that distortion will increase infinitely as the length of the contextual stimulus increases, which is obviously invalid. Another example is provided by a variant of Ponzo's pattern shown in Figure 2C. Such a pattern contains one half of a shrinkage form of a MüllerLyer pattern above the standard line and one half of an expansion form beneath the standard. An application of Formula 1 to this target would predict that no distortion should occur. Yet there is phenomenal expansion of the standard line, relative to that of the lower line. 
A



C



D



Figure 2. (A) A parallel-lines task. (B) A shrinkage form of the Müller-Lyer pattern as a composite of parallel lines. (C) A Ponzo pattern. (D) A minimum attentive field inscribed onto a Ponzo pattern.

\section{The Attentive Field}

A resolution of the problem posed by Figure $2 \mathrm{C}$ is achieved by recognizing that the stimulus field is not homogeneously processed in the way that a photograph represents all parts of a two-dimensional field with equal clarity. The fact is that the intentions of observers (to compare the two lines) determines where they will look. In order to carry out the task at all, it is necessary for the observer to process the end points of both standard and comparison lines. If a circle is inscribed through these four points, it will define a minimum attentive field (i.e., the smallest field that it is logically possible to postulate if the task is to be carried out at all). The center of this field is operationally defined as the midpoint between the two most distal points of the standard and comparison stimuli. $^{3}$ This definition is intended to apply equally to the case in which standard and comparison lines are collinear, as in the ambiguous Müller-Lyer or the Baldwin patterns (Pressey, 1974a; Pressey \& Wilson, 1980), or the case in which one line is directly above the other, as in the Ponzo illusion (Pressey, 1971). In this report, we will restrict ourselves to the latter configuration, versions of which are shown in Figure 2.

If a minimum field is drawn onto the Ponzo figure (as illustrated in Figure 2D), it can be seen that the expansion form of a Müller-Lyer pattern falls within the attentive field, and it will be most heavily weighted in the temporally integrated percept. The shrinkage form falls outside the field and does not contribute to the percept.

Care should be taken to distinguish between a minimum and an optimum field. The minimum field serves a useful theoretical and pedagogical purpose, but it is not intended to represent attention deployment of real observers. It is almost certain that attention is cast beyond the minimum field; this is represented by constructing circles that are larger than the minimum one. These circles represent optimum fields (i.e., sizes of fields that must be postulated in order to predict real performance).

In integrative field theory, position within the attentive field is one variable that modifies the degree of distortion in a parallel-lines task such as the one shown in Figure 3. The short standard line in the upper part of the display $\left(L_{s}\right)$ is judged in relation to the comparison line beneath it. The contribution of the contextual magnitude $\left(L_{c}\right)$ is calculated by measuring the distance from the center of attention to the tips of the contextual line $\left(D_{c}\right)$ and then relating this distance to the radius ( $r$ ) of a hypothetical circular field. Thus, the probability that the contextual line contributes to the judgment of the standard line is given by

$$
1-\frac{D_{c}}{r}
$$

In simulating actual experiments, several values of $r$ are sampled, because $r$ is presumed to be an organismic variable that reflects a particular style of deploying attention across a stimulus field. In most simulations, five radii of attentive field (ranging from 66 to $106 \mathrm{~mm}$ in steps of $10 \mathrm{~mm}$ ) are sampled. These radii are directly related to the parameters of the experimental targets as defined in the caption of Figure 3 . However, where $L_{s}$, or the distance between $L_{s}$ and the comparison line, varies, then the values of $r$ are multiplied by a ratio of the new minimum field to the original minimum field (Pressey \& Di Lollo, 1978). This point is clarified later when the experiments by Larsen, Garn, and Fritsch (1989) are simulated.

\section{The Interactive Field}

The attentive field is insufficient to account for several nuances in empirical trends, and it is necessary to postulate that the distance between $L_{c}$ and $L_{s}$ per se plays a role in effecting distortion (Pressey \& Murray, 1976). A second field, called the interactive field, is proposed. Because, as yet, there is no distinction made between various kinds of distances between contours (i.e., lateral vs. range differences), the center of this field is located at a tip of the standard line, as shown in Figure 3. The interactive field also specifies the probability that $L_{c}$ is sampled during the judgment of $L_{s}$, and it is derived by the formula

$$
1-\frac{D_{c}^{\prime}}{r^{\prime}}
$$

where $D_{c}^{\prime}$ is the distance between the tip of $L_{s}$ to the tip of $L_{c}$ and $r^{\prime}$ is the radius of the hypothetical interactive field.

Like the attentive field, it is assumed that the size of the interactive field reflects individual differences in perceptual style (in this case, in spatial discriminability), so that individuals with small fields are considered to be 


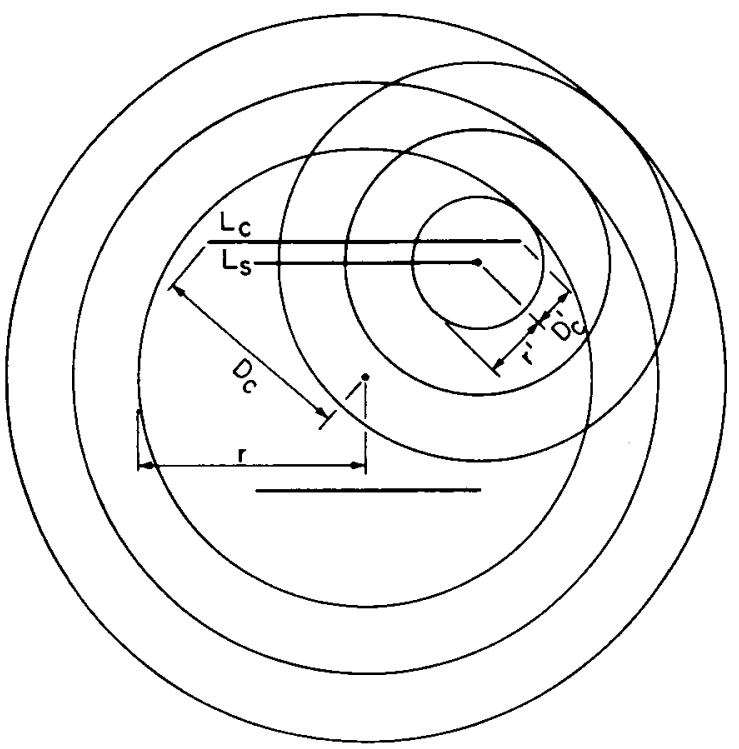

Figure 3. The parallel-lines display upon which the quantitative version of integrative field theory is based. $L$, is the standard line (which, in most cases, is $50 \mathrm{~mm}$ long), and $L_{c}$ is the contextual line. The comparison line is located $100 \mathrm{~mm}$ below the standard line and, on the average, appears directly below it. The large circles represent attentive fields of varying size. The center of these fields is depicted by the small dot, which is the midpoint between the most extreme points of the standard and comparison lines. $D_{c}$ is the distance from the center of the attentive field to the most distal point of the contextual line, and $r$ is the distance from the center of the attentive field to the edge of one hypothetical field. In most simulations, $r$ varied from 66 to $106 \mathrm{~mm}$ in steps of $10 \mathrm{~mm}$. The small circles represent interactive fields of varying size. The solid dot at the tip of the standard line is the center of these fields, which, in most simulations, varied from 10 to $40 \mathrm{~mm}$ in steps of $10 \mathrm{~mm}$. $D_{c}^{\prime}$ is the distance from the center of the interactive field to the most distal point of the contextual line, and $r^{\prime}$ is the radius of the smallest hypothetical interactive field.

highly discriminative whereas those with large fields are considered to be poorly discriminative. In simulations using the standard target described in Figure 3, four sizes of field with radii varying from 10 to $40 \mathrm{~mm}$, in steps of $10 \mathrm{~mm}$, are sampled.

When contributions of both the attentive field and the interactive field are considered simultaneously, the formula for calculating the distortion of $L_{s}$ is

$$
I=\left(1-\frac{D_{c}}{r}\right)\left(1-\frac{D_{c}^{\prime}}{r^{\prime}}\right)\left(L_{c}-L_{s}\right) \text {. }
$$

However, Formula 4 applies only to the case in which there is one contextual line, as in the parallel-lines illusion. Where complicated patterns such as the Müller-Lyer are used, these patterns are converted to a series of parallel lines, as shown in Figure 2B. A new value of $I$ (illusion) is calculated, and it represents a weighted average of all the contextual lines so sampled. The formula is given by

$$
I=\frac{1}{N} \sum_{j=1}^{N}\left(1-\frac{D_{c j}}{r}\right)\left(1-\frac{D_{c j}^{\prime}}{r^{\prime}}\right)\left(L_{c}-L_{s}\right),
$$

where $D_{c j}$ is the distance from the center of the attentive field to the most distant point of contextual magnitude $j$, $r$ is the radius of the attentive field, $D_{c j}^{\prime}$ is the distance from the tip of the standard line to the tip of contextual magnitude $j, r^{\prime}$ is the radius of the interactive field, and $N$ is the total number of contextual magnitudes sampled by this estimation procedure, all of which fall entirely within both the attentive and the interactive fields.

A computer program was developed using the standard experimental arrangement shown in Figure 3. This program permits a Müller-Lyer illusion to be predicted with variations in (1) the length of the standard line, (2) the orientation of the fin, (3) the angle of the fin, (4) the length of the fin, (5) the gap between the shaft and fin, (6) the distance between standard and comparison lines, (7) the size of the hypothetical attentive field, (8) the size of the hypothetical interactive field, and (9) any combination of the above eight variables.

In simulations, each predicted mean is the average of 20 separate estimates that result from sampling combinations of five attentive and four interactive fields. On the assumption that these 20 scores model individual differences in processing information, the standard deviations of these theoretical scores provide estimates of the standard deviations of real data (Pressey \& Murray, 1976).

\section{The Larsen-Garn Task}

Larsen and Garn (1988) measured distortion of an expansion form of Müller-Lyer's figure with a shrinkage form (or vice versa). The shafts of the two forms were located one above the other, and fins were removed either from between the two shafts or from outside the shafts. They found that removing the internal fins reduced distortion to the greatest degree and that removing the external fins had the least effect in altering distortion. These results are explained readily on the basis of an attentive field as proposed by integrative field theory. The fins that are closest to the center of the field will be most heavily weighted in the formation of the percept. The fins that are outside the shafts are far from the center of the field, they will be marginally processed, and they will contribute little to the percept.

The Larsen and Garn (1988) experiment was simulated with a quantitative version of integrative field theory (Pressey \& Kersten, 1989), and it was found that the predicted pattern of results was similar to the obtained pattern (Pearson's $r=.81$ ). Since then, Larsen and her associates have replicated the original study and introduced a methological change, the results of which highlight a weakness in integrative field theory.

\section{Simulating Experiments by Larsen et al. (1989)}

Larsen et al. (1989) used the targets employed in an earlier study (Larsen \& Garn, 1988) but asked observers to judge the shrinkage and expansion forms separately. According to integrative field theory (which assumes observers to be rational, intentional systems), the new instructions will produce a different attentive field, one in 


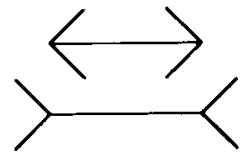

$\mathbf{A}+\mathbf{B}$

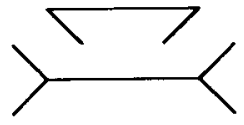

$\mathbf{C}+\mathbf{D}$

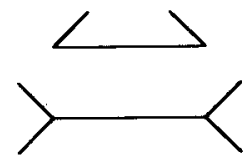

$E+F$

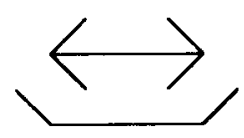

$\mathbf{G}+\mathbf{H}$

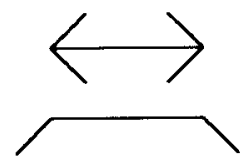

$\mathbf{I}+\mathbf{J}$
Figure 4. Versions of Müller-Lyer patterns used by Larsen and Garn (1988). The second letter in each pair refers to the case in which positions of the upper and lower targets are reversed.

which the center corresponds to the midpoint of the standard shaft. This proposal conforms to the definition provided by Jordan and Randall (1987) and defended (for certain conditions) by Pressey (1988). In the quantitative version of assimilation theory, the new field is simulated by allowing the distance between standard and comparison shafts to be equal to zero. Since the shaft was $53 \mathrm{~mm}$ long, the minimum field had a radius of $26.5 \mathrm{~mm}$; in the simulation of the experiment by Larsen et al. (1989), five radii, ranging from 26.5 to $76.5 \mathrm{~mm}$ in steps of $10 \mathrm{~mm}$, were sampled. As in previous simulations (e.g., Pressey, Di Lollo, \& Tait, 1977), the values of the interactive field varied from 10 to $40 \mathrm{~mm}$ in steps of $10 \mathrm{~mm}$. The predicted scores were as follows: targets $A+B=5.32 \mathrm{~mm}$, $C+D=3.54 \mathrm{~mm}, E+F=3.54 \mathrm{~mm}, \mathrm{G}+\mathrm{H}=4.28 \mathrm{~mm}$, and $\mathrm{I}+\mathrm{J}=4.28 \mathrm{~mm}$. The respective means obtained by Larsen et al. (1989) were 10.72, 9.80, 9.88, 8.22, and $8.06 \mathrm{~mm}$. Pearson's correlation coefficient was .21 . However, this value does not properly represent the goodness of fit between the two patterns because, in both cases, targets $A+B$ yielded the highest value, and $C+D$ was not different from $\mathrm{E}+\mathrm{F}$. Also $\mathrm{G}+\mathrm{H}$ was not different from $\mathrm{I}+\mathrm{J}$ in either set of scores. The main difficulty is that the theory predicts that removing the shrinkage fins $(C, D$, $E$, and $F$ ) should have a greater effect than removing the expansion fins ( $G, H, I$, and $J$ ), a result that is contradicted not only by Larsen et al., but by dozens of studies since Binet (1895) first reported an asymmetry in shrinkage and expansion forms of Müller-Lyer patterns.

It is possible that the difficulty described above stems from the fact that integrative field theory does not properly describe how attentive fields are linked to the stimulus array. Because, in the initial stages, the theory was fun- damentally a rational model, it was necessary to define the field only in terms of focal and comparison stimuli. Allowing the field to vary as a function of contextual stimuli, for example, would have made it a post hoc enterprise. Moreover, when empirical estimates of the sizes of fields were obtained (Pressey \& Murray, 1976), these values were used repeatedly in subsequent simulations (Pressey et al., 1977; Pressey \& Wilson, 1980) in order to maintain theoretical integrity.

However, it is becoming clear that the attentive field may be altered by the size of contextual, as well as focal, stimuli. For example, in a recent simulation of Holding's pattern that induces a positional shift (Pressey \& Smith Martin, 1990), it was found that the predicted function relating fin length to distortion was flatter than the obtained function. This means that the theory overestimates distortion for small fins and underestimates distortion for large fins. Therefore, it is possible that the radii of the hypothetical fields are too large for the small contextual fins and too small for the large fins. In order to assess this hypothesis, two routines were added to the computer program that generates quantitative predictions of distortion based on integrative field theory. The first addition simulated the Larsen-Garn task in which predictions for the five pairs of targets were generated using the standard interactive fields. All possible combinations of attentive field size were assigned separately to shrinkage and expansion forms. The radii of the attentive fields ranged from 26.5 to $76.5 \mathrm{~mm}$ in steps of $10 \mathrm{~mm}$, which yielded 36 predicted values for each of the five targets.

The second routine was a correlation program in which each of 36 patterns of predicted scores was correlated with the single pattern of obtained scores. The results, shown in Figure 5, are unequivocal. For shrinkage forms, the degree of fit (measured with Pearson's $r$ ) is best with the smallest attentive field size, and the degree of fit declines rapidly with increases in hypothetical size. On the other hand, for expansion forms, degree of fit increases with an increase in field size, and the best fit is obtained with the largest sampled size. These results indicate that the original lack of relationship that was found between predictions from integrative field theory and data from the Larsen et al. (1989) study was due to the fact that equal sizes of attentional fields were assigned to both the shrinkage and the expansion forms of Müller-Lyer figures.

Because conclusions drawn from Figure 5 were so clear, the remaining two experiments conducted by Larsen and her associates were simulated. In both cases, the best fit was provided by the smallest attentive field for the shrinkage form and the quality of fit declined with increases in field size. Conversely, for the expansion form, a field that was larger than the minimum size provided the best fit.

The conclusion that size of attentive field is related to type of Müller-Lyer target is supported not only by group trends but also by individual scores. Larsen kindly provided these scores, and each observer's pattern was subjected to a best-fit solution. The one cell in the correla- 


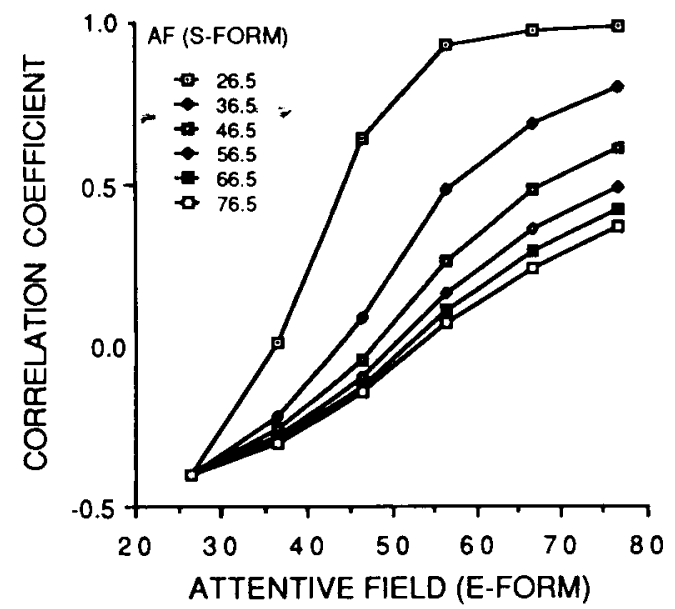

Figure 5. Correlations between simulated and real data as a joint function of size of hypothetical attentive fields for shrinkage (S-) and expansion (E-) forms of Müller-Lyer's illusion.

tion matrix that provided the largest value was selected, and the corresponding attentive fields for the shrinkage and expansion forms were recorded. There were $69 \mathrm{ob}-$ servers in the three studies; of these, $65.2 \%(z=2.53$, $p<.05)$ displayed patterns that required the attentive field to be greater for the expansion form than for the shrinkage form. There were 12 observers with equal attentive fields and 12 who exhibited a reversed pattern. Another encouraging result was that, in the main, it was possible to locate a single cell in the matrix of correlations that provided a good fit between predicted and observed data. The median correlation coefficient for the 69 observers was .86 .

The data from the Larsen et al. (1989) study were subjected to one additional test. A program was devised to provide a best-fit solution using the four interactive field sizes as a variable. The attentive fields were those that had been found to provide a best fit to the empirical data. The results of this simulation strongly supported the conclusion that, unlike attentive fields, interactive fields did not vary as a function of the shrinkage and expansion forms of the Müller-Lyer pattern.

On the whole, the simulations seem to indicate that attentive field size is related not only to focal but also to contextual stimuli and that, therefore, a circle provides an unnecessarily crude approximation of the perimeters of attentive fields. Perhaps a better visual representation (and one that can still be modeled by the mathematics of integrative field theory) is illustrated in Figure 6A. An alternative model is provided in Figure $6 \mathrm{~B}$, in which the center of the cone-shaped probability function is shifted closer to the large target. The present quantitative version of integrative field theory can simulate such a central shift, but because there is no evidence, at this point, to help us decide between these alternatives, the simulation procedure suggested in Figure 6A will be followed here.

\section{EXPERIMENT 1}

If attentive fields are related to characteristics of the target, then it should be possible to provide more information on how attention is deployed by varying those characteristics. Several variables, including size of the standard stimulus, size of the contextual stimulus, and distance between focal stimuli, have the potential of providing some insights.

Evidence on the manner in which attentive fields vary as a function of distance between focal stimuli already exists. Pressey and Di Lollo (1978) measured apparent shrinkage in a Müller-Lyer pattern as a joint function of gap between shaft and fins and of perpendicular distance between standard and comparison lines. These functions seemed to be properly simulated by allowing the attentive field to increase as a constant ratio of distance between shafts and by allowing interactive fields to remain the same. Experiment 1 was designed to confirm the empirical data and the theoretical interpretation provided by Pressey and Di Lollo. A task similar to the one used by Larsen and Garn (1988; see Figure 4) was employed.

\section{Method}

Subjects. Ten women and 2 men from the University of Manitoba participated as part of an introductory psychology course requirement. Only those with good vision, with or without glasses, were asked to volunteer.

Apparatus. All visual displays were shown on a black-and-white Electrohome monitor in which 100 pixels yielded a horizontal line of approximately $46 \mathrm{~mm}$. Three sets of 10 targets similar to those shown in Figure 4 were employed, in which each of the two horizontal shafts was $46 \mathrm{~mm}$ long; the fins were $10 \mathrm{~mm}$ long, and the angle between fins was $105^{\circ}$. The three sets differed in the perpendicular distances between shafts, which were 25,50 , and $75 \mathrm{~mm}$. The figure

A

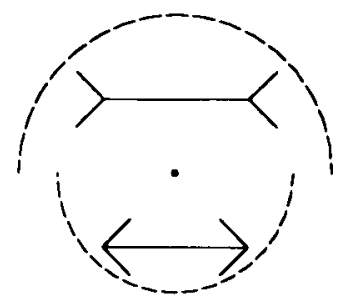

$\mathbf{B}$

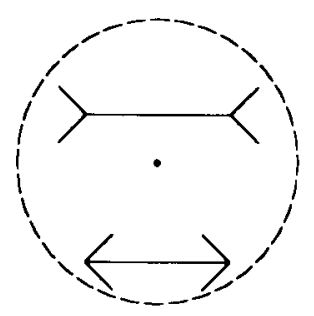

Figure 6. Two representations of attentive fields that conform to simulations of real data. 
at the bottom of the display was adjustable by having the computer replot the figure as it symmetrically changed the length of the shaft. Increments or decrements in shaft size and changes of the entire display were controlled by three buttons on an external response box. A pause was inserted after each plot of the comparison target in order to present perceptually distinct steps during adjustment.

The generation and randomization of targets, response acquisition, and scoring functions were all controlled by a computer. Testing was conducted in moderate illumination. An adjustable chinrest was located at the edge of a table so that an observer's line of vision was perpendicular to, and approximately $45 \mathrm{~cm}$ away from, the screen.

Procedure. After the observers were comfortably seated, a target from the appropriate set was randomly selected and displayed on the screen. The two horizontal shafts were pointed out, and the experimenter stated that they were physically equal but appeared of different length because of the fins that were attached to the ends of the shafts. Depending on which form of the Müller-Lyer figure was the comparison figure, the appropriate response button was pressed, and repeated plotting of the figure ensued. The observers were asked to note that, as plotting occurred, the two horizontal shafts began to appear equal in size. They were told that their task was to adjust the bottom figure until its shaft appeared equal in size to the one above it. It was emphasized that the observer was to respond to the apparent size of the shaft and not to perform the task by dropping imaginary perpendicular lines from the ends of the shafts. When instructions were understood, a new random sequence was generated and the task was completed in a self-paced manner.

The order of presenting the three sets of targets (which varied in distance between shafts) was counterbalanced between subjects, and the individual order was assigned as the subject appeared for testing. An interval of approximately 2 min separated each session, and the entire task was completed in about $10 \mathrm{~min}$.

\section{Results and Discussion}

Scores from targets $\mathrm{A}+\mathrm{B}, \mathrm{C}+\mathrm{D}, \mathrm{E}+\mathrm{F}, \mathrm{G}+\mathrm{H}$, and $\mathrm{I}+\mathrm{J}$ were summed and divided by 2 to obtain a single score for each pair. The scores were subjected to a $5 \times 3$ withinsubject analysis of variance (ANOVA) in which there were five types of Müller-Lyer targets and three distances $(25,50$, and $75 \mathrm{~mm})$ between shafts. The type of target $[F(4,44)=16.3, p<.05]$ and distance between shafts $[F(2,22)=8.08, p<.05]$ were statistically significant, but the interaction was not $[F(8,88)=1.31]$. The results, shown in Figure 7, substantiate the conclusion reached by Larsen and Garn (1988) and Larsen et al. (1989) that, in a compound version of a Müller-Lyer figure in which horizontal shafts are directly one above the other, shrinkage fins between shafts contribute most to the total effect. The shrinkage fins outside the shafts are least effective in producing distortion.

The fact that the degree of distortion increased with an increase in the distance between shafts supports earlier findings by Pressey and Di Lollo (1978), who reported that increasing the distance between focal and comparison shafts increased expansion of a focal shaft. Because these investigators found that their data could be simulated by increasing sizes of the hypothetical attentive fields (while keeping the sizes of the interactive fields the same), the data from Experiment 1 were subjected to a best-fit solution as outlined above. That is, correlation coefficients

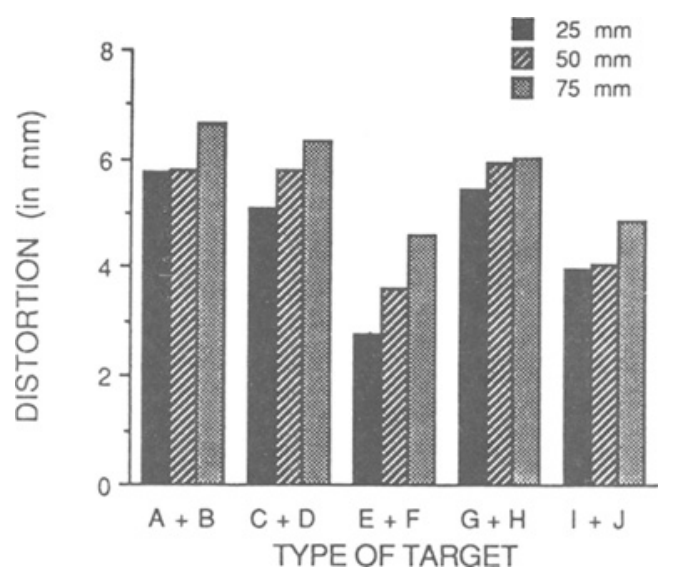

Figure 7. Distortions of Müller-Lyer targets as a function of distance between shafts.

were calculated between predicted and obtained scores under many combinations of sizes of attentive fields assigned separately to shrinkage and expansion forms of the illusion. The single cell in the matrix that yielded the highest coefficient was selected. Initially, it was intended to provide a best fit for each observer and then average across observers. However, the inferred values were not normally distributed, and the occasional extreme score skewed the measure of central tendency. Therefore, scores from all subjects were averaged and the best-fit solution was applied to the group means. The results, shown in Figure 8, indicate that the sizes of attentive fields necessary to provide the best fit increased with an increase in the distance between shafts. Thus, these results validate the simulation procedure used earlier by Pressey and Di Lollo (1978). The second point of interest in Figure 8 is that the hypothetical attentive fields for the shrinkage form were consistently smaller than those for the expansion form, confirming the conclusion reached in simulating data by Larsen et al. (1989).

\section{EXPERIMENT 2}

The purpose of Experiment 2 was to determine how attentive field size is related to target size when target size is varied by increasing the size of the standard line.

\section{Method}

Subjects. Fourteen women and 8 men from the University of Manitoba volunteered. Only those with good vision, with or without glasses, were asked to participate, and all were rewarded with partial credit toward their final grade in introductory psychology.

Apparatus and Procedure. All aspects of the experimental conditions were identical to those used in Experiment 1, except that only the one distance of $50 \mathrm{~mm}$ between shafts was selected and five shaft sizes $(27.6,36.8,46.0,55.2$, and $64.4 \mathrm{~mm})$ were employed. This resulted in a $5 \times 5$ within-subject design in which there were five types of targets and five sizes of shafts. The order of presenting the five lengths of shaft was randomized for each observer; targets within each level of shaft size were randomized separately for each observer. 


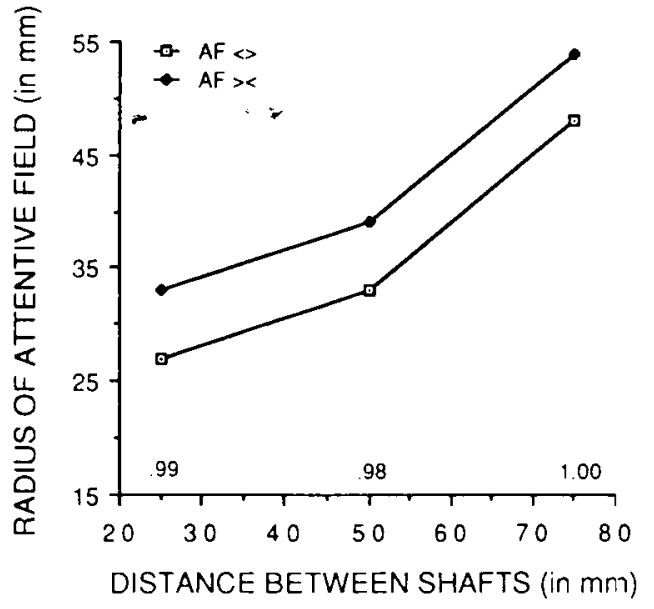

Figure 8. Radii of hypothetical attentive fields as a joint function of distance between shafts and forms of Müller-Lyer's illusion. The numbers above the abscissa are correlation coefficients that represent the best fit between theoretical and real data.

\section{Results and Discussion}

Scores were calculated as they were in Experiment 1, and they were subjected to a $5 \times 5$ within-subject ANOVA. Type of target $[F(4,84)=42.3]$, shaft size $[F(4,84)=$ 2.59 ], and the interaction between target and shaft size $[F(16,336)=4.23]$ were all significant beyond the .05 level of confidence. Figure 9 shows that, with the exception of targets $E+F$, the relationship between size of shaft and distortion approximated an inverted- $U$ function, which is reminiscent of the nonmontonic function that is reported when the shaft is constant but length of fin is increased (e.g., Heymans, 1896; Lewis, 1909; Restle \& Decker, 1977). The fins were of a constant size in Experiment 2, which means that the relative length of fin may be the important variable.

Theoretical functions derived from integrative field theory were calculated for a large number of combinations of hypothetical attentive fields assigned independently to shrinkage and expansion forms of the Müller-Lyer configuration. Each of these theoretical patterns was correlated with the average pattern of the group and, by a method of successive approximations, the optimum sizes of attentive field for the shrinkage and the expansion forms were selected. This procedure was repeated for the five sizes of shaft. The sizes of field so selected are shown in Figure 10. This figure shows that, again, an attentive field that was smaller for the shrinkage form than for the expansion form was required to provide the best fit at all sizes of shaft length. Because Pressey and Di Lollo (1978) and Jordan and English (1989) have proposed that the size of attentive fields changes as a constant ratio of the focal stimulus or the entire target, the optimum fields shown in Figure 10 were divided by the minimum size as defined by integrative field theory and illustrated in Figure 2D. The results, shown in Figure 11, seem unequivocal; the optimum size of the shrinkage form does appear to be a constant ratio of shaft (or target) size, but, for the expansion form, the ratio is highest for the smallest shaft and then it declines with increases in shaft size. This seems to mean that, for small objects, attention may be deployed beyond the boundaries of that object, whereas, for large objects, attention is captured by the boundaries. In other words, there may be a kind of "central tendency" operating in the manner in which attention is deployed in the visual field.

If the conclusions about attentive field size derived from Figure 11 are correct, it would be predicted that the asymmetry between shrinkage and expansion forms that has been reported repeatedly in the literature must be qualified. Since Binet's time (1895), investigators have reported that the expansion form of Müller-Lyer's configuration produces a greater degree of distortion than does the shrinkage form. From the results of this experiment, one would expect that shaft size would strongly modify that asymmetry. To our knowledge, only one study has measured the expansion and shrinkage forms separately as a function of shaft size. Bayer and Pressey (1972) showed that the proportional shrinkage effect did not vary as a function of shaft size, but the proportional expansion effect declined sharply as size of shaft increased.

\section{EXPERIMENT 3}

Experiment 3 was concerned with establishing how, according to integrative field theory, attentive fields vary as a function of contextual features by varying the length of fins in the complete and amputated Müller-Lyer configurations shown in Figure 4.

\section{Method}

Seven women and 11 men at the University of Manitoba were rewarded with partial credit toward their final grade in introductory psychology for participating in this experiment. The apparatus, procedure, and scoring of responses were identical to those used in

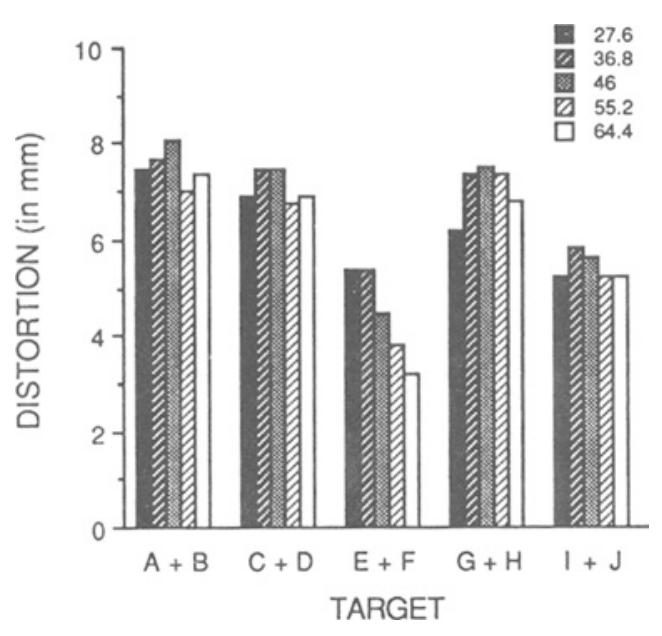

Figure 9. Distortions in Müller-Lyer tasks as a function of size of shan. 


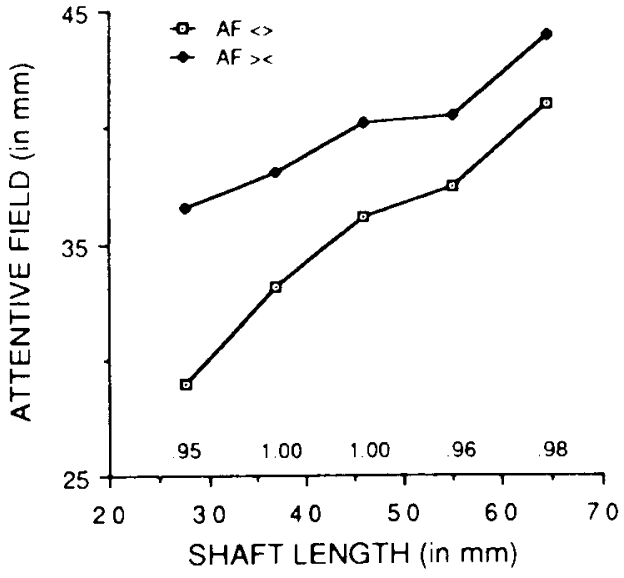

Figure 10. Radii of hypothetical attentive fields in shrinkage and expansion forms of Müller-Lyer's pattern as a function of length of standard shaft. The mumbers above the abscissa are corretation coeffcients that represent the best fit between theoretical and real data.

Experiment 2, except that size of fin replaced size of shaft as one of the independent variables. A $5 \times 6$ within-subject design was employed in which there were five types of Müller-Lyer targets and six levels of fin length. These varied from 5 to $30 \mathrm{~mm}$ in steps of $5 \mathrm{~mm}$.

\section{Results and Discussion}

A $5 \times 6$ within-subject ANOVA was conducted. Type of target $[F(4,68)=38.6]$, fin length $[F(5,85)=17.34]$, and the interaction $[F(20,340)=2.60]$ were significant beyond the .05 level of confidence. Figure 12 shows that, generally speaking, degree of distortion varies as an inverted-U function of fin length, which substantiates much of the previous research on this variable (e.g., Restle \& Decker, 1977). However, the significant interaction effect indicates that the exact shape of the function is related to the type of target that is employed. Optimal sizes of attentive fields were inferred in the same manner as in Experiments 1 and 2. The results are shown in Figure 13. The optimal attentive field for the shrinkage form of Müller-Lyer's illusion may decline somewhat as fin length increases, but the optimal size for the expansion form increases monotonically with an increase in fin length. However, it should be noted that there is no reversal in the optimal size when the fins are very large, thus failing to mimic the inverted- $U$ functions shown in Figure 12. This fact may be important in light of a recent hypothesis about attentive fields. Pressey and Smith Martin (1990) argued that

the attentive field might well increase as the outward pointing fins increase. In other words, increasing fin length can 'draw' attention well beyond the standard line which would result in a larger field as compared to an equivalent condition in which the fins are oriented toward the shaft. Furthermore, it is plausible to argue that as the outward pointing fins become very large, at some point they would fail to attract attention and field size would collapse back to the typical size employed by the observer. (p. 53)
Since no such reversal is evident in Figure 13, some other factor may be responsible for the inverted-U relationship between distortion and fin length. Jordan and his colleagues (Jordan \& English, 1989; Jordan \& Randall, 1987; Jordan \& Schiano, 1986) have proposed bidirectional fields in which contrast, rather than assimilation, operates at the periphery. It may be that the tips of very large fins fall within such contrast field and thus reduce the size of the assimilative effect.

As a matter of curiosity, the attentive fields derived from integrative field theory were inscribed onto the respective expansion forms of Müller-Lyer targets in the manner illustrated in Figure 14. The results are striking. The optimum size of the attentive field increases with increases in fin length, but the optimum fields incorporate different ratios of the external fin. The ratios for the 5-, $10-, 15-, 20-, 25-$, and $30-\mathrm{mm}$ fins are approximately $45 \%, 90 \%, 100 \%, 95 \%, 88 \%$, and $73 \%$. This pattern of ratios is clearly an inverted- $U$ function and may be the reason why Müller-Lyer's illusion varies as an inverted- $U$ function of fin length. Yet it is not clear why reducing the proportional length of fin that falls within the attentive field should reduce the magnitude of distortion, since the contextual lengths that do fall within the fields continue to increase in size as fin length increases. Thus, the effect of increasing fin length on Müller-Lyer's illusion continues to defy explanation; however, the relationship shown in Figure 14 should be pursued.

\section{EXPERIMENT 4}

Results from Experiments 1-3 indicate that attentive field size (as inferred from integrative field theory) varies as a function of the overall size of the stimulus display in a complicated, but coherent, manner. It is also possible to alter stimulus size by changing viewing distance. In such a case, the distal stimuli remain the same, but the

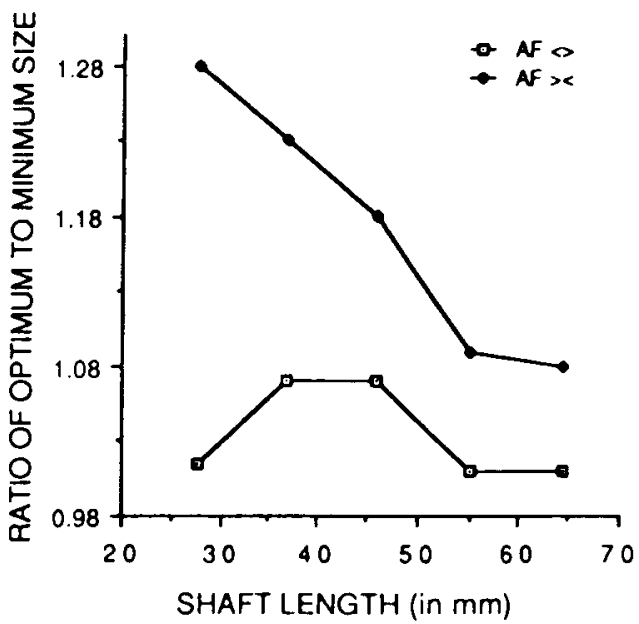

Figure 11. Ratios of optimum to minimum size of attentive fields in shrinkage and expansion forms of Müller-Lyer's pattern as a function of length of standard shan. 


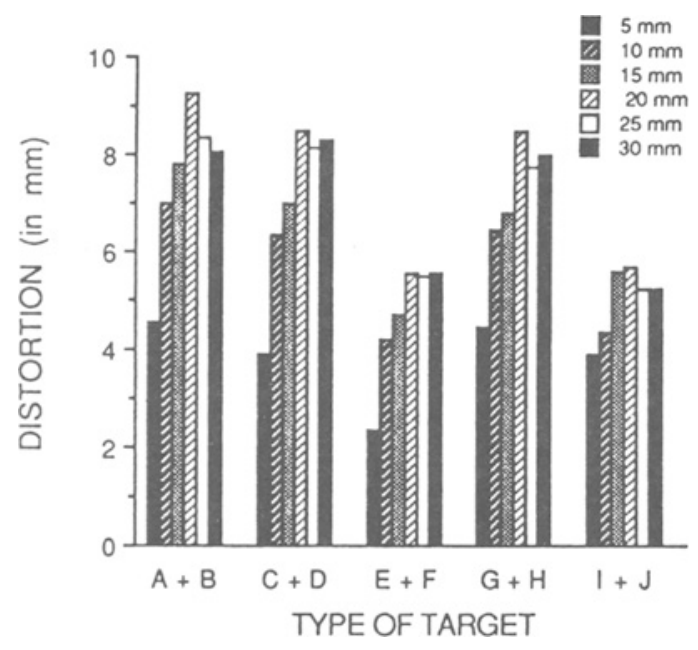

Figure 12. Distortions in Müller-Lyer tasks as a function of length of fin.

proximal (retinal size) is inversely related to viewing distance. The object of Experiment 4 was to determine whether attentive field size is related to the size of the object or to the size of the retinal image.

The second objective was a methodological one. In Experiments $1-3$, the bottom figure was always the adjusted figure. The effect of position of the adjustment figure was assessed by providing measurements with both the upper and the lower figures in the display.

\section{Method}

Fourteen male and 10 female students from an introductory psychology course at the University of Manitoba were rewarded with partial credit toward their final grade for participating. The apparatus, procedure, and scoring of responses were identical to those used in Experiments 1-3, with the following exceptions. First, no chinrest was employed; the three viewing distances $(45,90$, and $180 \mathrm{~cm}$ ) were measured by means of a demarcated string that was fastened to the monitor. Second, an additional program was written that was similar to the one used in Experiment 3, except that the size of the standard shaft was always $46.0 \mathrm{~mm}$, and the upper figure in the display was the adjusting (comparison) figure.

A $5 \times 3 \times 2$ mixed design was employed, in which there were five types of target, three viewing distances, and two positions of the adjusting figure. Position was balanced between subjects. Order of presenting viewing distances was completely counterbalanced between subjects and between adjusting positions.

\section{Results and Discussion}

An ANOVA for a mixed design showed that type of target $[F(4,88)=46.5, p<.05]$ was significant but that the effect of viewing distance was not $[F(2,44)=2.80$, $p>.05]$. None of the remaining values of $F$ approached statistical significance. Figure 15 shows the familiar pattern relating distortion to type of target, and it shows a very small, but seemingly consistent, decline in distortion with viewing distance.

Optimal attentive fields were inferred by the computational procedures described earlier; these are shown as a function of viewing distance in Figure 16. What is striking is not the small rise in the sizes of attentive fields but rather their constancy across viewing distances. After all, the maximum change in the radii of optimal fields across viewing distance was only $3 \mathrm{~mm}$, which means that the size of the attentive field seems to be related to the size of the object and not to the size of the retinal image. This conclusion is supported by Jordan and Schiano (1986), who found that assimilation and contrast are affected by relative, rather than absolute, spatial characteristics of the stimuli. To use the spotlight metaphor, the attentive field is not a constant cone whose distal diameter increases with distance. Rather, it is a cone whose visual angle is determined by the distal object. In other words, attentive fields display constancy by being tied to the object in the same way that apparent size, shape, and brightness are linked to characteristics of objects rather than to the characteristics of retinal representations.

\section{GENERAL DISCUSSION}

\section{Attentive Fields and Object Size}

The most general conclusion that these experiments support is that spatial deployment of attention is not limited to the focal stimulus as integrative field theory had initially proposed. Rather, it seems that the size (and possibly the shape) of the attentive field is closely tied to the size and shape of the object that is being apprehended. Thus, for example, with the shrinkage form of MüllerLyer's target, the attentive field remains nearly the same size as length of fin increases. This could be due to the fact that the size of the object that the shrinkage form represents does not change markedly. It is true that the dimension that is orthogonal to the shaft increases, but the dimension defined by the shaft itself is unchanged.

The expansion form of Müller-Lyer's configuration could represent a different set of conditions. With in-

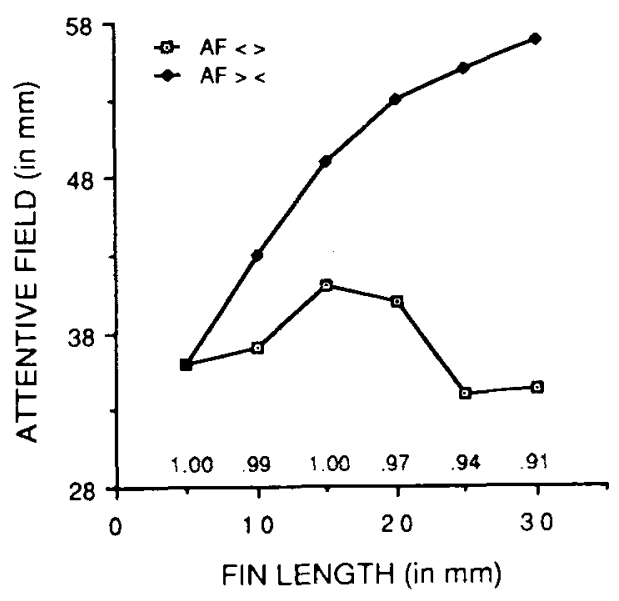

Figure 13. Radii of hypothetical attentive fields in shrinkage and expansion forms of Müller-Lyer's pattern as a function of length of fin. The numbers above the abscissa are correlation coefincients that represent the best fit between theoretical and real data. 


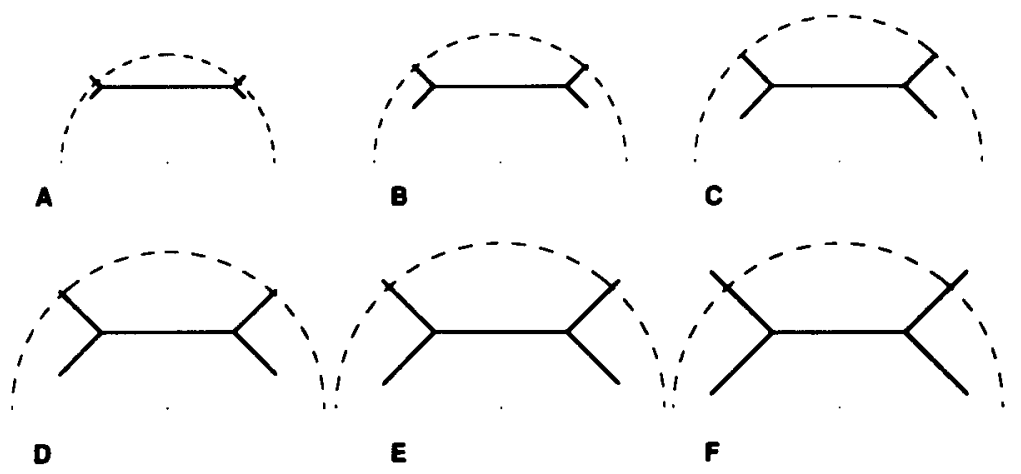

Figure 14. Representations of attentive field size as a function of length of fins in expansion forms of Müller-Lyer figures.

creases in fin length, the dimension orthogonal to the shaft increases and so does the dimension that is parallel to the shaft. In other words, in the shrinkage form, increasing fin size could represent a single object that is rotated in the $y-z$ plane, whereas the expansion form must represent objects of different sizes that are so rotated. Consequently, if the attentive field is related to the size of the object (as the data on viewing distance seem to suggest), then the fact that attentive fields change with changes in the expansion figures but remain relatively constant with the shrinkage figures would make sense. In addition, the fact that optimal attentive fields increase with increases in size of shaft is also consistent with the notion that attention deployment is tied to the object character of MüllerLyer figures.

Our findings are supported by LaBerge's (1983) research. He used a probe technique to measure reaction time in a categorization task and concluded that "the spotlight width in the letter tasks is one letter space, and the spotlight width in the [five-letter] word task is typically five spaces" (p. 371). Thus, it appears that spatial distribution of attention is remarkably similar in temporally constrained and temporally extended tasks. In addition, it seems that breadth of attention deployment is determined by the size of the object that is to be processed. A similar conclusion was reached by Podgorny and Shepard (1983), who showed that "compactness" of figures affected how attention was distributed. Such compactness may well be identical to the dimension of target size used in our experimental tasks.

\section{Spatial Distribution of Attention and Asymmetry in Distortion}

The results of these experiments provide us with a better understanding of a problem that was noted as early as 1895 by Binet. He observed that the shrinkage form of Müller-Lyer's figure induced a smaller degree of distortion than did the expansion form. Subsequent investigators have verified this asymmetry and have discovered that the two forms behave differently when such variables as angle of fins, length of fins, and intertip distance are varied (Adam \& Bateman, 1980; Day \& Dickinson, 1976;
Greist-Bousquet \& Schiffman, 1981; Sekuler \& Erlebacher, 1971). Because of these facts, many investigators have concluded that the two forms are "fundamentally different," but the exact nature of this fundamental difference has never been specified. The answer, according to the results of the experiments reported here, is as follows: The two forms are different because the shrinkage form is treated as if it represents a single entity whose size is equal to the size of the shaft. However, the expansion form is treated as an object whose size is determined by the tips of the fins. This could be due to a primitive figure-ground articulation whose function is to establish the outer boundary of the object, much as the outer boundary is phenomenally established in the Mackworth effect shown in Figure 1. Also, Marr's (1982) notion of a "primal sketch" would be consistent with such an interpretation. Or it could be that the expansion form represents an object in three-dimensional perspective, as proposed by Thiéry (1896), Tausch (1954), Gregory (1963), and Warren and Bashford (1977). But whatever the reason, the perceiver attends to the shrinkage and expansion forms in a different way. Attention is determined by the

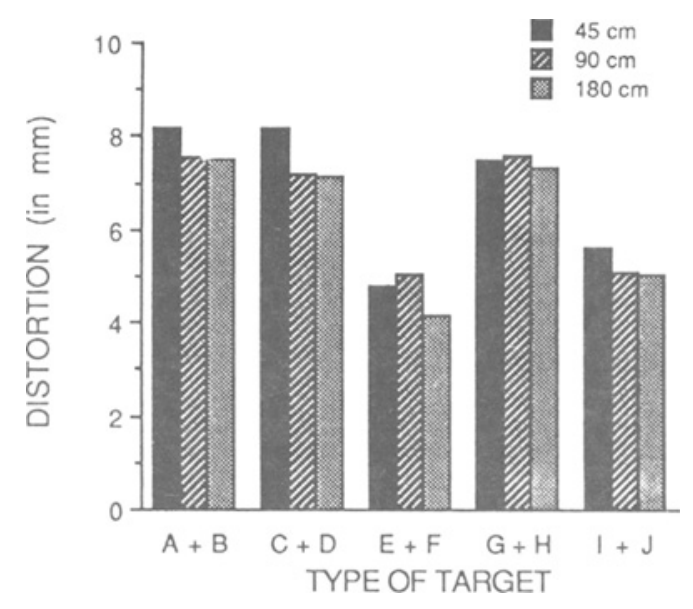

Figure 15. Distortions in Mutler-Lyer tasks as a function of viewing distance. 


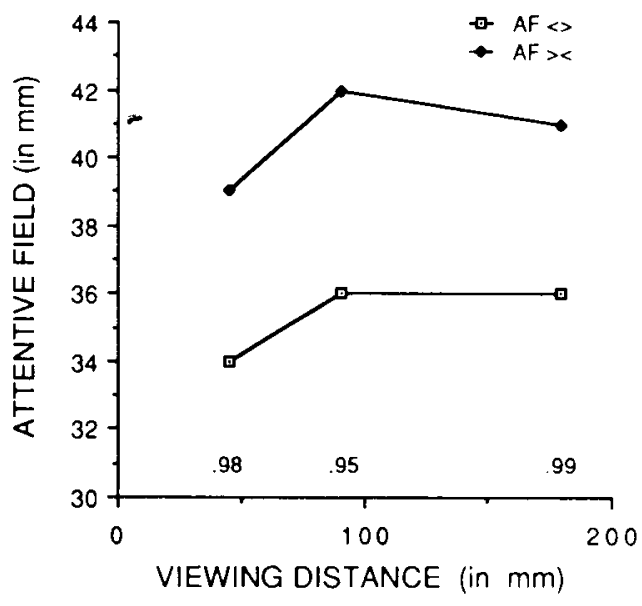

Figure 16. Radii of hypothetical attentive fields as a function of viewing distance in shrinkage and expansion forms of Müller-Lyer's patterns. The numbers above the abscissa are correlation coeficients that represent the best fit between theoretical and real data.

tips of the shaft in the shrinkage form and by the tips of the wings in the expansion form. Given this fact, and given the remaining ideas associated with integrative field theory, a more detailed qualitative answer to the problem of asymmetry is possible. Recall that integrative field theory proposes that perception of a standard magnitude is influenced by three attributes of the contextual line. One is the size difference between the two lines. The second is the spatial separation between the standard and contextual lines. The third is the location that the contextual lines occupy within an attentive field. The degree of distortion increases as size difference increases, but it decreases as the spatial separation increases, and it decreases as the contextual line moves away from the center of attention. It is this last proposition that is crucial for understanding the many differences that occur between the two forms of illusion. Thus, for example, Adam and Bateman (1980) and Sekuler and Erlebacher (1971) reported that only the shrinkage illusion varied with size difference as measured by intertip distance (ITD). Degree of distortion was not significantly related to ITD in the configuration that yields expansion.

According to integrative field theory, the interaction between ITD and form of illusion would be expected. In the shrinkage form, changes in ITD are produced primarily by moving the fins toward the center of attention. Therefore, when distortion is plotted as a function of ITD, a pronounced trend will be observed. However, in the expansion form, increases in ITD are produced by moving the fins away from the center of attention. Therefore, increases in ITD are accompanied by decreases in the probability that ITD will be effective, which could result in a less dramatic (and possibly nonsignificant) relationship between illusion and ITD.

\section{Individual Differences}

When a quantitative version of integrative field theory was first written (Pressey, Butchard, \& Scrivner, 1971), several values of attentive field size were selected to represent individual differences in attention deployment. Borrowing from work by Klein and his associates (e.g., Gardner, Holzman, Klein, Linton, \& Spence, 1959), it was proposed that individuals varied in their typical breadth of deploying attention in a visual display. Those who focused attention in a small region confined to the relevant stimuli (and thus attenuated irrelevant peripheral information) were modeled by small attentive fields. Such individuals would display small distortions that were calculated by applying Weighting Formula 2 (given above). On the other hand, individuals who typically deployed attention broadly across a display were represented by large attentive fields and, hence, large distortions. The addition of this dimension to the model allowed variances to be predicted, and it was shown that the fit between predicted and obtained variances was quite close (Pressey et al., 1971; Pressey \& Murray, 1976). However, no additional tests of the presumed involvement of this perceptualcognitive style were provided.

The present study allows us to pursue the issue of stable individual differences in more detail. Specifically, best-fit solutions of attentive field size were obtained for the two forms of illusion not only for group trends, but also for each individual pattern. These sizes of field were then correlated with the size of the full version of MüllerLyer's pattern (i.e., targets A+B). Data from Larsen's (Larsen \& Garn, 1988; Larsen, Garn, \& Fritsch, 1989) three experiments and from the experiments reported in the present study were analyzed. Since our experiments involved repeated measures, a single score for each experiment was obtained by averaging correlation coefficients across conditions. This procedure yielded seven estimates of goodness of fit between obtained and predicted functions. Pearson's $r$ for the attentive field for the shrinkage form of Müller-Lyer targets and size of distortion was -.09 , which was not statistically significant. But the average coefficient of +.19 for the expansion form was significantly different from the expected value of zero $[t(6)$ $=3.00, p<.05]$. Inspection of all scores revealed that expansion targets with relatively long fins yielded higher coefficients. This result is consistent with the point made by Girgus and Coren (1987) that the role of organismic variables is best assessed by targets that induce large distortions. However, it appears that, at best, only about 5\% to $10 \%$ of the variance is attributable to stable individual differences in attention deployment.

\section{Concluding Remarks}

In the development of integrative field theory, it has always been assumed that attention is focused primarily on standard (or target) stimuli and that contextual stimuli are processed inadvertently. The results of these experiments are instructive because they appear to indicate that attention is "captured" by the configuration of the entire target in which the edge is highly processed, perhaps in order to segregate a figure from its ground. This means that much research on, say, the Müller-Lyer illusion may be misguided because of the failure to recognize that 
closure is involved as the perceiver strives to apprehend the object character of the figure. Piaget (1961) is one of the few who seems to have recognized the role of closure, because he considered Müller-Lyer figures to be "trapezia" and "lozenges," and he conducted studies on forms displaying varying degrees of completeness. Others, such as Gillam (1971), Tausch (1954), and Warren and Bashford (1977) have linked geometric illusions to everyday perception. Generally, however, the study of such distortions from the premise that perceivers are intentional or representational systems (Dennett, 1978) has not been adequately pursued.

\section{REFERENCES}

ADAm, J., \& BAtEman, L. (1980). Control stimuli in investigations of the acute-angled and obtuse-angled Müller-Lyer illusions. Perception, 9, 467-474.

Bayer, C. A., Pressey, A. W. (1972). Geometric illusions as a function of pigmentation of the Fundus oculi and target size. Psychonomic Science, 26, 77-79.

Binet, A. (1895). La mèsure des illusions visuelles chez les enfants Revue Philosophique, 40, 11-25.

BroAdBent, D. E. (1982). Task combination and selective intake of information. Acta Psychologica, 50, 253-290.

DAy, R. H., Dickinson, R. G. (1976). Apparent length of the arms of acute and obtuse angles, and the components of the Müller-Lyer illusion. Australian Journal of Psychology, 28, 137-148.

DenNeTt, D. (1978). Brainstorms: Philosophical essays on mind and psychology. Cambridge: MIT/Bradford.

Eriksen, B. A., ERIKSEN, C. W. (1974). Effects of noise letters upon the identification of a target letter in a nonsearch task. Perception \& Psychophysics, 16, 143-149.

Eriksen, C. W., Goettl, B., St. James, J. D., Fournier, L. R. (1989). Processing redundant signals: Coactivation, divided aftention, or what? Perception \& Psychophysics, 45, 356-370.

ErIKSEN, C. W., ST. JAmEs, J. D. (1986). Visual attention within and around the field of focal attention: A zoom lens model. Perception \& Psychophysics, 40, 225-240.

ERIKSEN, C. W., YEH, Y. (1985). Allocation of attention in the visual field. Joumal of Experimental Psychology: Human Perception \& Performance, 11. 583-597.

Gardner, R. W., Holzman, P. S., Klein, G. S., Linton, H. B., - SPENCE, D. P. (1959). Cognitive control: A study of individual consistencies on cognitive behaviour. Psychological Issues, 1(4), 1-186.

GAtTI, S. V., EgETh, H. E. (1978). Failure of spatial selectivity in vision. Bulletin of the Psychonomic Society, 11, 181-184.

GiLLAM, B. (1971). A depth processing theory of the Poggendorff illusion. Perception \& Psychophysics, 10, 211-216.

Girgus, J. S., COREN, S. (1987). The interaction between stimulus variations and age trends in the Poggendorff illusion. Perception \& Psychophysics, 41, 60-66.

Gogel, W. C., SharkeY, T. J. (1989). Measuring attention using induced motion. Perception, 18, 303-320.

GREGoRy, R. L. (1963). Distortion of visual space as inappropriate constancy scaling. Nature, 199, 678-680.

Greist-Bousquet, S., Schiffman, H. R. (1981). The many illusions of the Müller-Lyer: Comparisons of the wings-in and wingsout illusions and manipulations of standard and dot forms. Perception, 10, 147-154.

Heymans, G. (1896). Quantitative Untersuchungen über das "optische Paradoxon." Zeitschrift fur Psychologie, 9. 211-255.

Jones, R. S. (1982). Physics as metaphor. New York: New American Library.

JoRdAN, K., \& ENGLISH, P. W. (1989). Simultaneous sampling and length contrast. Perception \& Psychophysics, 46, 546-554.
JORDAN, K., RANDALL, J. (1987). The effects of framing ratio and oblique length on Ponzo illusion magnitude. Perception \& Psychophysics, 41, 435-439.

JoRdAN, K., SCHIANO, D. J. (1986). Serial processing and the paralkellines illusion: Length contrast through relative spatial separation of contours. Perception \& Psychophysics, 40, 384-390.

LABERGE, D. (1983). The spatial extent of attention to letters and words. Joumal of Experimental Psychology: Human Perception \& Peformance, 9. 371-379.

LARSEN, J. D., GARN, N. K. (1988). Wings in the intershaft space contribute to the Mueller-Lyer illusion. Perceptual \& Motor Stills. 67, 831-834.

Larsen, J. D., GArn, N. K., Fritsch, T. (1989). Effect of intershaft wing removal on the Mueller-Lyer illusion as a function of judgment task. Perceptual \& Motor Skills, 68, 699-704.

LEWIS, E. O. (1909). Confluxion and contrast effects in the MüllerLyer illusion. British Joumal of Psychology, 3. 21-42.

Mackworth, N. H. (1965). Visual noise causes tunnel vision. Psychonomic Science, 3, 67-68.

MARR, D. (1982). Vision. San Francisco: W. M. Freeman

Merjkle, P. M., Gorewich, N. J. (1979). Spetial selectivity in vision: Field size depends upon noise size. Bulletin of the Psychonomic Sociery, 14, 343-346.

Piaget, J. (1961). Les mécanismes perceptifs. Paris: Presses Universitaires de Gravé.

Podgorny, P., Shepard, R. N. (1983). Distribution of visual attention over space. Joumal of Experimental Psychology: Human Perception \& Performance, 9, 380-393.

Posner, M. I., Snyder, C. R. R., Davioson, B. J. (1980). Attention and the detection of signals. Journal of Experimental Psychology: General, 109, 160-174.

Pressey, A. W. (1967). A theory of the Mueller-Lyer illusion. Perceprual \& Motor Skills, 25, 569-572.

Pressey, A. W. (1971). An extension of assimilation theory to illusions of size, area, and direction. Perception \& Psychophysics, 9. 172-176.

Pressey, A. W. (1972). The assimilation theory of geometric illusions: An additional postulate. Perception \& Psychophysics, 11, 28-30.

Pressey, A. W. (1974a). Effect of size of angle on the ambiguous Müller-Lyer illusion. Acta Psychologica, 38, 401-404.

Pressey, A. W. (1974b). Evidence for the role of attentive fields in the perception of illusions. Quanerly Joumal of Experimental Psychology, 26, 464-471

Pressey, A. W. (1979). Are black circles attentive fields? A reply to Bross, Blair and Longtin. Perceprion, 8, 237-238.

Pressey, A. W. (1982). The parallel lines illusion as a measure of cognitive style (Report). Winnipeg: Manitoba Mental Health Research Foundation.

Pressey, A. W. (1988). A comment on "The effects of framing ratio and oblique length on Ponzo illusion magnitude." Perception \& Psychophysics, 43, 201-202.

Pressey, A. W., Butcharo, N., \& Scruvner, L. (1971). Assimilation theory and the Ponzo illusion: Quantitative predictions. Canadian Joumal of Psychology, 25, 486-497.

Pressey, A. W., Di Lollo, V. (1978). Effects of distance between standard and comparison lines on the Müller-Lyer illusion. Perception \& Psychophysics, 24, 415-419.

Pressey, A. W., Di Lollo, V., TAIT, R. W. (1977). Effects of gap size between shaft and fins and angle of fins on the Müller-Lyer illusion. Perception, 6, 435-439.

Pressey, A. W., Kersten, B. (1989). Atention and the Müller-Lyer illusion: Simulation of an experiment by Larsen and Garn. Perceptual \& Motor Skills, 68, 1323-1329.

Pressey, A. W., \& Murray, R. (1976). Further developments in the assimilation theory of geometric illusions: The adjacency principle. Perception \& Psychophysics, 19, 536-544.

Pressey, A. . Smith Martin, N. (1990). The effects of varying fins in Müller-Lyer and Holding illusions. Psychological Research, 52. 46-53. 
Pressey, A. W., WiLson, A. E. (1980). Assimilation theory and the Baldwin illusion. Italian Journal of Psychology, 7, 65-73.

Pressey, A. W., Wilson, A. E., \& HarPer, D. W. (1980). Evidence for the role of attentive fields in masking. Perception, 9, 31-36.

Restle, F., \& Decker, J. (1977). Size of the Mueller-Lyer illusion as a function of its dimensions: Theory and data. Perception \& Psychophysics, 21, 489-503.

SANDERS, A. (1963). The selective process in the functional visual field. Soesterberg, The Netherlands: Institute for Perception, RVO-TNO.

Sekuler, R., Erlebacher, A. (1971). The two illusions of MüllerLyer: Confusion theory reexamined. American Journal of Psychology, 84, 477-486.

TAUSCH, R. (1954). Optische Täuschungen als artifizielle Effekte der Gestaltungsprozesse von Grössen-und Formenkonstanz in der natürlichen Raumwahrnehmung. Psychologische Forschung, 24, 299-348.

ThiẺry, A. (1896). Über geometrisch-optische Täuschungen. Philosophische Studien, 12, 67-126.

WARREN, R. M., BASHFORD, J. A. (1977). Müller-Lyer illusions: Their origin in processes facilitating object recognition. Perception, 6, 615-626.

WERTHEIMER, M. (1987). A brief history of psychology (3rd ed.). New York: Holt, Rinehart and Winston.

\section{NOTES}

1. Early in our work, we chose to use the phrase attentive field to differentiate it from notions proposed by Mackworth (1965) and Sanders
(1963). In this report, we have restricted the phrase to refer to a spatial region of a display that represents differing probabilities of being sampled during the performance of a task. The phrase antentional field refers to hypothetical mechanisms or processes; the phrase field of attention is intended to be a generic term that subsumes all discourse on spatial distribution of attention.

2. In the literature, this approach has been called Pressey's assimilation theory to differentiate it from numerous other explanations that appeal to assimilative-like processes to explain perceptual distortions. It is hoped that the phrase integrative field theory will replace the former label, because the phrase is both a more descriptive and a more accurate one.

3. This definition is fundamentally a rational one that allows the theory to be translated into mathematical form. Nevertheless, it is also plausible as a psychological proposition because, during a comparison, observers repeatedly attend first to one line and then to the other. Consequently, the center of the field could be most heavily represented with the result that centrally located information would be most heavily weighted in the formation of a percept.

(Manuscript received April 26, 1991; revision accepted for publication December 10, 1991.) 